\title{
Mesures des concentrations de stéroïdes et de $\beta$-agonistes dans des laits pour nourrissons
}

\author{
S Moslemi, P Silberzahn*, GE Séralini * \\ Laboratoire de biochimie et biologie moléculaire, EP 0009 CNRS, Irba, \\ université de Caen, 14032 Caen cedex, France
}

(Reçu le 22 novembre 1995 ; accepté le 24 juillet 1996)

\begin{abstract}
Résumé - Les concentrations de stéroïdes endogènes (progestérone, androstènedione, testostérone, estrone, estradiol et nortestostérone), celles d'un stéroïde xénobiotique (diéthylstilbestrol) et de $\beta$-agonistes (salbutamol et clenbutérol) ont été mesurées dans 12 laits différents reconstitués, commercialisés en poudres pour nourrissons. Les composés recherchés sont extraits sur des cartouches Sep-Pak $\mathrm{C}_{18}$. Après une série de délipidations, hydrolyses enzymatiques, extractions puis purifications sur colonnes de gel de silice, ces molécules ont été quantifiées par dosage radio-immunologique. Les mesures effectuées dans des échantillons de $3 \mathrm{~g}$ de poudres de laits reconstitués dans $20 \mathrm{~mL}$ d'eau / sulfate de triéthylamine $(1: 1, \mathrm{v} / \mathrm{v})$ indiquent que trois des hormones stéroïdes présentent des concentrations relativement élevées par rapport à l'ensemble dosé : la progestérone $(7,48 \pm 4,31 \mathrm{ng} / \mathrm{mL})$, l'androstènedione $(0,61 \pm 0,26 \mathrm{ng} / \mathrm{mL})$ et l'estrone $(0,07 \pm 0,05 \mathrm{ng} / \mathrm{mL})$. La somme des concentrations de l'estradiol, qui est l'estrogène physiologiquement le plus actif, de la testostérone et de la nortestostérone atteint au total $0,05 \pm 0,03 \mathrm{ng} / \mathrm{mL}$ seulement. Les concentrations mesurées, notamment pour la progestérone et les estrogènes, correspondent aux concentrations physiologiques du lait de vache. Les résultats montrant la non-détection du diéthylstilbestrol (la limite de détection est de $6,25 \mathrm{pg} / \mathrm{mL}$ de lait dans nos conditions) confirment que cette molécule n'apparaît pas être employée dans l'élevage des vaches laitières à un taux qui permettrait de la déceler dans les laits maternisés. Les faibles concentrations mesurées pour les $\beta$-agonistes correspondent à la limite de détection de ces composés, qui est de l'ordre de $12,5 \mathrm{pg} / \mathrm{mL}$. D'autre part, nos dosages font apparaître une différence notable, en particulier au niveau de la concentration physiologique de progestérone, entre les laits maternisés et le lait de femme.
\end{abstract}

dosage radio-immunologique / stéroïde / anabolisant / $\beta$-agoniste / lait maternisé

Summary - Measurements of steroid and $\beta$-agonist concentrations in infant milks. The concentrations of endogenous steroids (progesterone, androstenedione, testosterone, estrone, estradiol and nortestosterone), of a xenobiotic steroid (diethylstilbestrol) and of $\beta$-agonists (salbutamol and clenbuterol) were measured in 12 different infant milks, commercialized as powders. The samples were first chromatographed on Sep-Pak $C_{18}$ cartridges. The compounds were assessed by radioimmunoassays after delipidations, enzymatic hydrolyses, extractions and purifications on silica gel columns. Among the compounds analyzed from $3 \mathrm{~g}$ of powder milk reconstituted to $20 \mathrm{~mL}$ of water/triethylamine

\footnotetext{
* Adresse actuelle : Inspection générale de l'Agriculture, 35, rue Saint-Dominique, 75007 Paris, France.

** Correspondance et tirés à part.
} 
sulfate $(1: 1, \mathrm{v} / \mathrm{v})$, only progesterone $(7.48 \pm 4.31 \mathrm{ng} / \mathrm{mL})$, androstenedione $(0.61 \pm 0.26 \mathrm{ng} / \mathrm{mL})$ and estrone $(0.07 \pm 0.05 \mathrm{ng} / \mathrm{mL})$ reached relatively elevated levels. The sum of estradiol concentrations, which is physiologically the most active estrogen, plus those of testosterone and nortestosterone, attained altogether only $0.05 \pm 0.03 \mathrm{ng} / \mathrm{mL}$. The measured concentrations, especially for progesterone and estrogens, correspond to the physiological levels published for cow's milk. The diethylstilbestrol was not detectable in the samples (its detection limit is $6.25 \mathrm{pg} / \mathrm{mL}$ in our conditions), and thus this molecule does not appear to be used in cow breeding actually, at least at a level allowing its detection in infant milk. The values obtained for $\beta$-agonists were at the detection limit which was $12.5 \mathrm{pg} / \mathrm{mL}$. Moreover, our assays evidence a noticeable difference for progesterone levels between commercialized infant milks and the maternal milk.

radioimmunoassay / steroid / anabolic agent / $\beta$-agonist / infant milk

\section{INTRODUCTION}

Stéroïdes et $\beta$-agonistes sont parfois utilisés en production animale au cours des traitements médicaux ou frauduleusement comme facteurs de croissance. L'utilisation illégale des stéroïdes comme anabolisants est bien connue. Les $\beta$-agonistes sont employés pour la thérapie chez l'homme et l'animal, notamment dans le traitement des affections pulmonaires, mais ils sont aussi connus comme facteurs de répartition (agents de répartition lipido-protéique) et pourraient donc être utilisés en tant que tels en production animale. L'ensemble de ces molécules et de leurs dérivés est actif par voie orale, ils peuvent donc être ajoutés aux rations des animaux. Leur utilisation comme facteurs de croissance s'est dernièrement considérablement accrue, à différentes périodes selon les pays.

Le contrôle des résidus dans les aliments destinés aux enfants constitue une garantie de qualité des produits nécessaire et réclamée par les consommateurs. Le lait est l'aliment essentiel pour le nouveau-né, et il occupe pour une partie importante de la population la place principale en alimentation, au moins jusqu'à l'âge de 2 ans. Le contenu hormonal anormal du lait peut avoir des effets significatifs sur la physiologie des nourrissons et des enfants. II est donc primordial de connaître le contenu hormonal du lait en général, et du lait maternisé en particulier, reconstitué pour les nourrissons à partir des poudres de laits de vaches traitées ou non.

Les dosages effectués dans ce travail permettent de le découvrir, et de comparer les concentrations hormonales au lait de femme. Nous avons donc mesuré les taux d'hormones stéroïdes et de $\beta$-agonistes dans 12 laits commercialisés en poudre destinés à l'alimentation des nourrissons.

\section{MATÉRIELS ET MÉTHODES}

Les poudres de lait utilisées sont des échantillons anonymes de différents laits de commerce qui nous ont été fournis par le Syndicat français des aliments de l'enfance et de la diététique (Paris, France), à reconstituer à raison de $3 \mathrm{~g} / 20 \mathrm{~mL}$. Les stéroïdes non marqués proviennent de Sigma (Saint-Quentin-Fallavier, France), le diéthylstilbestrol (DES) de Steraloids (Wilton, NH, États-Unis). Les stéroïdes radiomarqués proviennent d'Amersham (Les Ulis, France) : $\left[1,2,6,7-{ }^{3} \mathrm{H}\right]$ androstènedione $(3330$ $\mathrm{GBq} / \mathrm{mmol}),\left[2,4,6,7^{-3} \mathrm{H}\right]$ estradiol $(3293-4070$ $\mathrm{GBq} / \mathrm{mmol}),\left[2,4,6,7-{ }^{3} \mathrm{H}\right]$ estrone (3515 $\mathrm{GBq} / \mathrm{mmol}),\left[1,2,6,7-{ }^{3} \mathrm{H}\right]$ progestérone (3108 $\mathrm{GBq} / \mathrm{mmol}),\left[1,2,6,7-{ }^{3} \mathrm{H}\right]$ testostérone $(3478$ $\mathrm{GBq} / \mathrm{mmol}), 19-\left[6,7(\mathrm{n})-{ }^{3} \mathrm{H}\right]$ nortestostérone ( $703 \mathrm{GBq} / \mathrm{mmol}$ ) et [monoéthyl- ${ }^{3} \mathrm{H}$ ] diéthylstilbestrol (2590-4440 $\mathrm{GBq} / \mathrm{mmol})$. Le laboratoire d'hormonologie (Marloie, Belgique) est le fournisseur de $\left[{ }^{3} \mathrm{H}\right]$ clenbutérol $(481 \mathrm{GBq} / \mathrm{mmol})$ et des anticorps spécifiques antinortestostérone (dilution finale utilisée 1/70 000), antidiéthylstilbestrol (utilisé au 1/200 000) et antisalbutamol (au $1 / 24000$ ), ainsi que des produits pour le dosage radio-immunologique (RIA) du clenbutérol. Les anticorps spécifiques antitestostérone, anti- 
androstènedione, antiestradiol, antiestrone et antiprogestérone viennent de bioMérieux, Charbonniẻres-les-Bains, France.

Ces anticorps ont été utilisés à la dilution finale 1/10 000. Waters (Millipore, Milford, MA, ÉtatsUnis) a fourni les cartouches Sep-Pak $C_{18}$. L'enzyme Hélicase provient d'IBF, Villeneuve-laGarenne, France.

\section{Reconstitution du lait en poudre}

Trois grammes de chaque poudre de lait (chacune correspondant à une marque déposée) sont solubilisés pour reconstitution dans $10 \mathrm{~mL}$ d'eau bidistillée (chauffée à $62-64^{\circ} \mathrm{C}$ ) et laissés à la température ambiante pendant 1 heure. La figure 1 résume les différentes étapes réalisées dans ce travail pour l'analyse des stéroïdes et des $\beta$-agonistes. Avant le passage sur les coIonnes Sep-Pak $C_{18}$, les échantillons sont dilués avec $10 \mathrm{~mL}$ de sulfate de triéthylamine $(0,5$ mol L-1, $\mathrm{pH} 7$,2) puis chauffés dans un bain d'eau à $64^{\circ} \mathrm{C}$ pendant 5 minutes (Axelson et Sahlberg, 1981 ; Sahlberg et Axelson, 1986).

\section{Extraction des stéroïdes et des $\beta$-agonistes sur Sep-Pak $C_{18}$}

L'efficacité des colonnes Sep-Pak $C_{18}$ pour extraire les stéroïdes du lait est bien décrite (Axelson et Sahlberg, 1981 ; Sahlberg et Axelson, 1986). L'utilisation du sulfate de triéthylamine et le passage de l'échantillon à chaud à travers le SepPak $\mathrm{C}_{18}$ sont deux conditions expérimentales indispensables pour minimiser les interactions stéroïdes-protéines et stéroïdes-lipides (Axelson et Sahlberg, 1981).

Avant de déposer l'échantillon à l'aide d'une seringue $(20 \mathrm{~mL})$, la cartouche de Sep-Pak $\mathrm{C}_{18}$ est activée avec $5 \mathrm{~mL}$ de méthanol, puis lavée avec $5 \mathrm{~mL}$ d'eau bidistillée $\left(64^{\circ} \mathrm{C}\right)$.

\section{Extraction des stéroïdes}

L'échantillon, chauffé à $64^{\circ} \mathrm{C}$, est partagé sur deux cartouches de Sep-Pak $C_{18}(10 \mathrm{~mL}$ par cartouche). Le dépôt d'échantillon sur la cartouche est réalisé à l'aide d'une seringue, avec un débit de $5 \mathrm{~mL} / \mathrm{min}$ environ. Les cartouches sont alors lavées avec $5 \mathrm{~mL}$ d'eau bidistillée $\left(64^{\circ} \mathrm{C}\right)$. Tous les stéroïdes (libres et conjugués) sont élués par $5 \mathrm{~mL}$ de méthanol, puis par $5 \mathrm{~mL}$ de méthanolchloroforme $(1: 1, v / v)$ à température ambiante. L'extrait est évaporé à sec à l'aide d'un évapo- rateur rotatif (Jouan RC 10.22, Saint-Herblain, France).

\section{Extraction des $\beta$-agonistes}

Le processus est identique à celui décrit pour l'extraction des stéroïdes, hormis le fait que les cartouches sont acidifiées avec $1 \mathrm{~mL}$ de $\mathrm{H}_{2} \mathrm{SO}_{4}\left(0,2 \mathrm{~mol} \mathrm{~L}^{-1}\right)$ avant l'élution des $\beta$ agonistes par $5 \mathrm{~mL}$ de méthanol. L'extrait est évaporé à sec.

\section{Délipidation des échantillons}

L'extrait sec est repris dans $5 \mathrm{~mL}$ de méthanol et laissé une nuit à $-30^{\circ} \mathrm{C}$ (ou $2-3$ heures à $-78^{\circ} \mathrm{C}$ ). Après une centrifugation à $800 \mathrm{~g}$ à $-4^{\circ} \mathrm{C}$ et pendant 15 minutes, la phase méthanolique de chaque extrait est transférée dans un autre tube, puis évaporée à sec.

\section{Hydrolyse enzymatique des stéroïdes conjugués}

L'extrait sec est repris dans $5 \mathrm{~mL}$ de tampon acétate $\left(0,2 \mathrm{~mol} \mathrm{~L}^{-1} \mathrm{pH} 4,8\right)$, puis additionné de $50 \mu \mathrm{L}$ d'Hélicase $\left(10^{6}\right.$ unités Fishman $/ 10 \mathrm{~mL}$ pour la $\beta$-glucuronidase et $10^{7}$ unités Roy $110 \mathrm{~mL}$ pour la sulfatase) et laissé une nuit à $37^{\circ} \mathrm{C}$. Les stéroïdes rendus libres sont extraits de l'hydrolysat refroidi successivement par $10 \mathrm{~mL}$ d'éther diéthylique, puis d'éther de pétrole et enfin d'acétate d'éthyle. En cas d'émulsion, 200-400 $\mu \mathrm{L}$ d'éthanol sont additionnés. Les phases organiques concernant chaque extraction sont regroupées dans un seul tube et évaporées à sec.

\section{Saponification}

Afin d'estimer une éventuelle présence de l'estradiol-17 $\beta$ sous forme d'esters, une saponification de la fraction non polaire écartée après délipidation a été réalisée. Pour ce faire, $3 \mathrm{~mL}$ de $\mathrm{KOH} 60 \% /$ éthanol $(90: 10, \mathrm{v} / \mathrm{v})$ ont été ajoutés à la phase lipidique et incubés 2 heures à $85^{\circ} \mathrm{C}$. Après avoir dilué avec $2 \mathrm{~mL}$ d'eau, le milieu non neutralisé a été extrait par $2 \times 5 \mathrm{~mL}$ d'acétate d'éthyle. L'extrait est ensuite évaporé à sec et traité comme les autres échantillons pour le dosage RIA. Le rendement d'extraction de cette étape a été évalué grâce à un traceur radioactif (estradiol-17ß) à $62,4 \pm 2,4 \%$ 
$3 \mathrm{~g}$ de poudre de lait

$+10 \mathrm{~mL}$ d'eau $\left(64^{\circ} \mathrm{C}\right)$ après $1 \mathrm{~h}$

$+10 \mathrm{~mL}$ triéthylamine-

sulfate $0,5 \mathrm{~mol} / \mathrm{L}\left(64^{\circ} \mathrm{C}\right) \mathrm{pH} 7,2$<smiles>C=C</smiles>

Sep-Pak C18

(10 $\mathrm{mL}$ par cartouche)

Cartouches acidifiées avec

$1 \mathrm{~mL}$ d'acide sulfurique $(0,2 \mathrm{~mol} / \mathrm{L})$ avant l'élution des $ß$-agonistes par $5 \mathrm{~mL}$ de méthanol<smiles>CC=CC</smiles>

L'extrait est évaporé à sec (Hélicase) toute la nuit<smiles>C[As]1CCC1</smiles>

Extraction des $B$-agonistes par $2 \times 10 \mathrm{~mL}$ d'acétate d'éthyle après avoir ajusté (pH 12-13,5)

le milieu avec $\mathrm{NaOH} 2 \mathrm{~N}$

$$
\mid
$$

L'extrait est évaporé à sec

RIA
Extraction des stéroïdes par $10 \mathrm{~mL}$ d'éther diéthylique, d'éther de pétrole et d'acétate d'éthyle

Hydrolyse enzymatique (Hélicase) toute la nuit à $37^{\circ} \mathrm{C}$
Elution des stéroiides

(libres et conjugués)

par $5 \mathrm{~mL}$ de méthanol et $5 \mathrm{~mL}$ de méthanol/chloroforme (1:1)

L'extrait est évaporé à sec

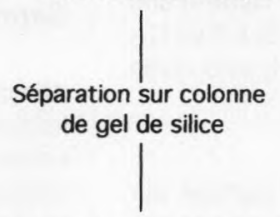

L'extrait est évaporé à sec

RIA
Saponification de la phase lipidique

Extraction par $2 \times 5 \mathrm{~mL}$ d'acétate d'éthyle

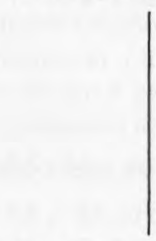

L'extrait est évaporé à sec

RIA estradiol

Fig 1. Différentes étapes utilisées pour l'analyse des stéroïdes et $\beta$-agonistes dans les laits pour nourrissons.

Different steps for steroid and $\beta$-agonist assays in infant milk samples. 
( $n=3)$ et pris en compte dans le calcul des résultats.

\section{Séparation des stéroïdes sur gel de silice}

Les colonnes sont préalablement préparées de la façon suivante : des pipettes en verre (Coming, New York, États-Unis) de $5 \mathrm{~mL}$, avec pour diamètre interne $6 \mathrm{~mm}$, sont munies d'un bouchon de coton hydrophile puis remplies par $2 \mathrm{~mL}$ de gel de silice 60 (Merck, Darmstadt, Allemagne) maintenu en suspension dans du toluène. Les extraits sont solubilisés dans $4 \times 100 \mu \mathrm{L}$ de toluène et appliqués puis élués par le mélange toluène / acétate d'éthyle $(85: 15, v / v)$. Les stéroïdes sont séparés grâce à cette technique.

\section{Purification des $\beta$-agonistes}

Après l'étape d'extraction sur Sep-Pak $C_{18}$, nous avons utilisé la technique de Girault et Fourtillan (1990) légèrement modifiée pour purifier les $\beta$ agonistes. Après délipidation, l'extrait sec est repris dans $1 \mathrm{~mL}$ d'eau bidistillée. On ajuste le $\mathrm{pH}$ à 4,8 à l'aide du $\mathrm{NaOH} 1 \mathrm{~N}$. L'hydrolyse enzymatique est réalisée comme précédemment. L'hydrolysat refroidi est centrifugé à $800 \mathrm{~g}$ pendant 10 minutes à $4^{\circ} \mathrm{C}$. La phase aqueuse " clarifiée " est récupérée dans un autre tube et extraite successivement par $10 \mathrm{~mL}$ d'éther diéthylique, puis d'éther de pétrole et enfin d'acétate d'éthyle. Après avoir ajusté le pH (à 12-13,5) de la phase aqueuse avec du $\mathrm{NaOH} 2 \mathrm{~N}$, les $\beta$-agonistes sont extraits par $2 \times 10 \mathrm{~mL}$ d'acétate d'éthyle. Les phases organiques sont évaporées à sec à l'aide de l'évaporateur rotatif.

\section{Rendements}

Les rendements après toutes les étapes décrites plus haut, ont été évalués par l'addition de standards radioactifs $(20 \quad 000-50000 \mathrm{cpm})$ à des échantillons. Les pourcentages de récupérations réalisées trois fois pour trois laits differents (sauf pour le diéthylstilbestrol et le clenbutérol) sont respectivement au final de : $25 \pm 4,6 \%$ (pour la progestérone), $61 \pm 12 \%$ (androstènedione), $45,5 \pm 2,3 \%$ (testostérone), $48,9 \pm 8,1 \%$ (nortestostérone), $60 \pm 7,5 \%$ (estrone), $45 \pm 8,7 \%$ (estradiol), $43 \%$ (diéthylstilbestrol) et $44 \%$ (clenbutérol).

\section{Dosage radio-immunologique (RIA) et spécificité}

Après l'incubation de l'extrait avec l'anticorps et l'homologue tritié à $37^{\circ} \mathrm{C}$ pendant 30 minutes (dosage par compétition), les stéroïdes libres non liés à l'anticorps sont adsorbés par $500 \mu \mathrm{L}$ de charbon-dextran $(0,268-0,0268 \%$, bioMérieux, Charbonnières-les-Bains, France) dilué dans du PBS, pour $300 \mu \mathrm{L}$ d'échantillon. La mesure de la radioactivité de la fraction liée à l'anticorps permet la détermination des concentrations du stéroïde recherché dans les échantillons, par référence à une courbe d'étalonnage réalisée avec des concentrations connues du stéroïde non marqué $(6,25-800 \mathrm{pg})$, des concentrations fixes du stéroïde marqué et de l'anticorps. Les comptages de radioactivité sont analysés par le programme Securia RIA (Packard, Rungis, France). La même méthode est utilisée pour le dosage des $\beta$-agonistes, avec de légères modifications. L'anticorps est incubé avec les différentes concentrations de la gamme ou avec les extraits à $4{ }^{\circ} \mathrm{C}$ pendant une nuit. On ajoute l'homologue tritié (dosage par déplacement) et on incube encore 2 heures à $4^{\circ} \mathrm{C}$. Après avoir additionné le charbon-dextran, on poursuit comme ci-dessus.

Pour éviter les réactions croisées, nous avons séparé les stéroïdes qui possèdent des structures voisines, par exemple la testostérone de l'androstènedione et l'estradiol de l'estrone. Le pourcentage des réactions croisées des différents anticorps est donné dans le tableau I, récapitulatif des précisions des fournisseurs. De plus, pour savoir si l'anticorps antiestradiol utilisé dans ce travail reconnaissait ou pas un phytoestrogène tel que l'équol, qui peut être présent dans le lait de vache (Barkley et al, 1985), des gammes d'étalonnages pour le dosage RIA ont été réalisées en présence d'anticorps antiestradiol, d'estradiol radiomarqué, d'estradiol non marqué $(6,2-400 \mathrm{pg} /$ tube) $)$ ou d'équol $(1,9-$ $500 \mathrm{ng} /$ tube).

\section{Sensibilité et précision}

La sensibilité du dosage RIA prise en compte pour établir les gammes standards ( $n=3$ à 5 ) est de $3,13 \mathrm{pg} / \mathrm{mL}$ de lait pour l'estradiol, la progestérone, l'estrone, l'androstènedione et de $6,25 \mathrm{pg} / \mathrm{mL}$ pour le diéthylstilbestrol, la testostérone, la nortestostérone, et de $12,5 \mathrm{pg} / \mathrm{mL}$ pour les $\beta$-agonistes. Afin d'estimer la variation de sensibilité pour les dosages, nous avons réalisé 
Tableau I. Pourcentage de réactions croisées avec différentes hormones pour les anticorps antistéroïdes utilisés.

Percentage of cross-reactions for the antisteroid antibodies used in RIA.

\begin{tabular}{|c|c|c|c|c|c|c|c|}
\hline \multirow[t]{2}{*}{ Stéroïdes } & \multicolumn{7}{|c|}{$\%$ de réactions croisées des anticorps antihormones pour $50 \%$ de liaison } \\
\hline & $E_{1}$ & $E_{2}$ & $T$ & $A$ & NT & $P_{4}$ & DES \\
\hline Estrone & 100 & 3,50 & $<0,01$ & $<0,01$ & $<0,1$ & $<0,01$ & $<0,1$ \\
\hline Estradiol $17 \beta$ & 1,0 & 100 & $<0,01$ & $<0,01$ & $<0,1$ & $<0,01$ & $<0,1$ \\
\hline Estradiol $17 \alpha$ & $<0,01$ & $<0,03$ & $<0,01$ & $<0,01$ & $<0,01$ & $<0,01$ & $<0,1$ \\
\hline Estriol & 0,04 & - & - & - & $<0,01$ & - & $<0,1$ \\
\hline Nortestostérone & 0,03 & $<0,01$ & 12,1 & 0,08 & 100 & - & - \\
\hline Norandrostènedione & 0,20 & $<0,01$ & 0,04 & 25,3 & 46,3 & - & - \\
\hline Norépitestostérone & - & - & - & - & 55 & - & - \\
\hline $\begin{array}{l}\text { Norandrostanedione } \\
3 \beta-17 \alpha \text {-diol - }\end{array}$ & - & - & - & - & 20,1 & - & - \\
\hline Norandrostane & - & - & - & - & 4,4 & - & - \\
\hline Testostérone & $<0,01$ & $<0,01$ & 100 & 0,36 & $<0,03$ & $<0,01$ & $<0,1$ \\
\hline Androstènedione & $<0,01$ & $<0,01$ & 1,90 & 100 & - & $<0,01$ & - \\
\hline Androstérone & - & - & - & - & $<0,02$ & - & - \\
\hline Epitestostérone & $<0,01$ & $<0,01$ & 0,02 & 0,06 & $<0,01$ & $<0,01$ & - \\
\hline Dihydrotestostérone & $<0,01$ & $<0,01$ & 50 & 0,05 & $<0,01$ & $<0,01$ & - \\
\hline Progestérone & $<0,01$ & $<0,01$ & 0,05 & - & $<0,04$ & 100 & - \\
\hline Diéthylstilbestrol & - & - & - & - & $<0,01$ & - & 100 \\
\hline
\end{tabular}

Les abréviations correspondent à celles de la figure 2. Ces valeurs ont été fournies par le Laboratoire d'hormonologie (Marloie, Belgique) pour NT et DES, et pour le reste par bioMérieux (Charbonnières-les-Bains, France).

Abbreviations for hormones are indicated in figure 2. These values were given by the Laboratoire d'Hormonologie (Marloie, Belgique) for NT and DES, and by bioMérieux (Charbonnières-les-Bains, France) for the others.

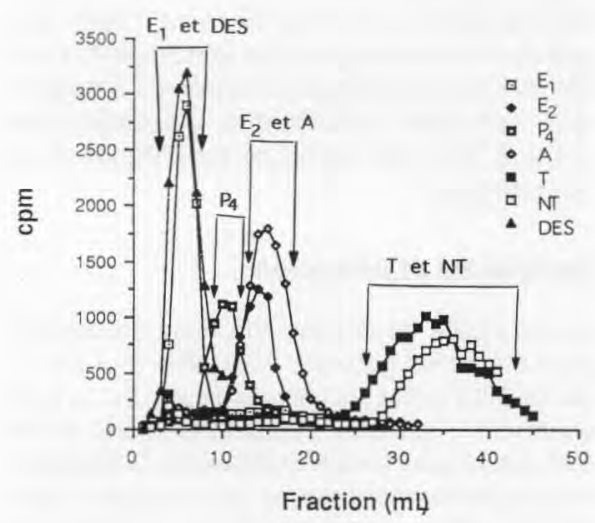

Fig 2. Profils d'élution des hormones dosées. Les séparations de l'estrone $\left(E_{1}\right)$, de l'estradiol $\left(E_{2}\right)$, de la progestérone $\left(P_{4}\right)$, de l'androstènedione (A), de la testostérone ( $T$ ), de la nortestostérone (NT) et du diéthylstilbestrol (DES) sont réalisées sur colonnes de gel de silice $(9,5 \times$ $0,6 \mathrm{~cm}$ ). Les stéroïdes sont élués par le mélange toluène / acétate d'éthyle $(85: 15$, v/v). Les flèches indiquent l'ensemble des fractions utilisées pour le dosage de chaque hormone.

Elution profiles for measured hormones. Separations of estrone $\left(E_{1}\right)$, estradiol $\left(E_{2}\right)$, progesterone $\left(P_{4}\right)$, androstenedione $(A)$, testosterone (T), nortestosterone (NT) and diethylstilbesterol (DES) were performed on silica gel columns $(9,5$ $x 0,6 \mathrm{~cm})$. Steroids were then eluted by toluene / ethyl acetate $(85: 15, v / v)$. The arrows indicate the pooled fractions used for radioimmunoassays. 
des tests de surcharge après évaporation des échantillons et avant le dosage RIA pour le clenbutérol (7,8-1000 pg/tube) et l'estradiol (3,1$400 \mathrm{pg} /$ tube), retrouvés en quantités très faibles ou non détectables dans les échantillons.

Pour la précision, la valeur de chaque concentration obtenue est la moyenne de deux expériences en double détermination. La déviation standard de la moyenne a été respectivement inférieure à 4,9\% (pour les $\beta$-agonistes), 2,7\% (estradiol), 8,9\% (progestérone), 4,1\% (estrone), 2,8\% (testostérone), 4,9\% (androstènedione) et $2,2 \%$ (nortestostérone).

\section{RÉSULTATS ET DISCUSSION}

La plupart des lipides non polaires est éliminée sur la colonne de Sep-Pak $\mathrm{C}_{18}$. Le reste des lipides est éliminé pendant la délipidation des échantillons avec le méthanol. Ces deux étapes d'élimination des lipides et de purification des stéroïdes sur colonnes de gel de silice (fig 2) sont primordiales pour minimiser les réactions croisées des anticorps antistéroïdes lors du dosage RIA. En effet, la remarquable sensibilité des dosages RIA est connue. Mais ce type de dosage peut donner des résultats par excès, notamment dans le cas des milieux non connus, ou lors de l'utilisation de milieux contenant un mélange de stéroïdes aux structures voisines. Ces conditions nous ont conduit à séparer les molécules avant le dosage proprement dit.

Les mesures effectuées dans des échantillons de $3 \mathrm{~g}$ de poudres de laits reconstitués dans $20 \mathrm{~mL}$ d'eau / sulfate de triéthylamine $(1: 1, v / v)$ indiquent que trois des hormones stéroïdes mesurées présentent les concentrations les plus élevées : la progestérone $(7,48 \pm 4,31 \mathrm{ng} / \mathrm{mL})$, l'androstènedione $(0,61 \pm 0,26 \mathrm{ng} / \mathrm{mL})$ et l'estrone $(0,07 \pm 0,05 \mathrm{ng} / \mathrm{mL})$. L'ensemble des concentrations de l'estradiol, qui est l'estrogène physiologiquement le plus actif, de la testostérone et de la nortestostérone ne dépasse pas $0,05 \pm 0,03 \mathrm{ng} / \mathrm{mL}$ (tableau II). Le diéthylstilbestrol et les $\beta$-agonistes ne sont pas détectés ou à la limite de détection.
Le contenu hormonal du lait varie continuellement suivant l'état physiologique de la vache. De plus, une vache laitière est en moyenne gestante 6 mois sur une durée d'allaitement de 9 mois. Nous comparons les valeurs obtenues dans ce travail, d'une part avec celles publiées pour le lait de vaches non traitées dans différentes conditions (gestation ou non, lait entier ou écrémé, etc), et d'autre part avec des valeurs obtenues pour le lait de femme sans traitement hormonal. L'ensemble des données est rassemblé dans le tableau III.

\section{La progestérone}

Chez la vache, les concentrations de progestérone dans le lait et dans le plasma varient selon l'état physiologique (estrus ou gestation), la nature du lait (entier ou écrémé) et le moment du prélèvement (matin ou après-midi). Les valeurs publiées varient aussi suivant la méthode employée pour la quantification de l'hormone. L'étude bibliographique fait état de concentrations allant de 2,4 à $5,1 \mathrm{ng} / \mathrm{mL}$ dans le lait des vaches non gestantes, et de 4,73 à $19,7 \mathrm{ng} / \mathrm{mL}$ chez les gestantes (Laing et Heap, 1971 ; Schiavo et al, 1975 ; Pope et al, 1976 ; Schemesh et al, 1978).

Dans tous les cas, de gestation ou non, la progestérone dans le lait entier peut être environ deux fois plus élevée que dans le plasma (Schiavo et al, 1975 ; Pope et al, 1976). Schemesh et al (1978) mesurent par RIA les concentrations de progestérone dans le lait entier, écrémé, et dans le plasma jugulaire chez des vaches gestantes, elles sont respectivement de 4,73, 2,63 et $2,9 \mathrm{ng} / \mathrm{mL}$. Cette concentration élevée de progestérone dans le lait par rapport au sang pourrait être due au fait que les glandes mammaires de vache contiennent l'enzyme nécessaire à la synthèse de la progestérone à partir de la prégnénolone ; la présence de la $3 \beta$-hydroxystéroïde déshydrogénase dans les glandes mammaires de chèvre a d'ailleurs été démon- 
Tableau II. Concentrations des stéroïdes $(\mathrm{ng} / \mathrm{mL})$ dans le lait reconstitué avec $3 \mathrm{~g}$ de poudre pour $20 \mathrm{~mL}$ d'eau / sulfate de triéthylamine $(1: 1, \mathrm{v} / \mathrm{v})$.

Steroid and $\beta$-agonist concentrations $(\mathrm{ng} / \mathrm{mL})$ in infant milk reconstituted $(3 \mathrm{~g} / 20 \mathrm{~mL}$ of water / triethylamine sulfate, $1: 1, v / v)$.

\begin{tabular}{|c|c|c|c|c|c|c|}
\hline \multirow[t]{2}{*}{ Échantillons } & \multicolumn{6}{|c|}{ ng par $\mathrm{mL}$ de lait reconstitué à $3 \mathrm{~g} / 20 \mathrm{~mL}$} \\
\hline & $\mathrm{P}_{4}$ & A & $E_{1}$ & $\mathrm{~T}$ & $E_{2}^{*}$ & NT \\
\hline 1 & 9,959 & 0,911 & 0,118 & 0,019 & ND & 0,008 \\
\hline 2 & 12,959 & 0,736 & 0,017 & 0,011 & ND & 0,007 \\
\hline 3 & 6,284 & 0,354 & 0,006 & 0,034 & 0,005 & ND \\
\hline 4 & 12,771 & 0,879 & 0,148 & 0,024 & 0,014 & ND \\
\hline 5 & 4,446 & 0,641 & 0,123 & 0,023 & 0,006 & 0,011 \\
\hline 6 & 15,959 & 0,633 & 0,013 & 0,072 & 0,009 & 0,008 \\
\hline 7 & 4,259 & 0,299 & 0,063 & 0,018 & ND & ND \\
\hline 8 & 3,321 & 0,418 & 0,024 & 0,015 & ND & 0,008 \\
\hline 9 & 8,121 & 0,474 & 0,063 & 0,059 & 0,003 & 0,010 \\
\hline 10 & 1,671 & 0,228 & 0,036 & 0,018 & ND & 0,010 \\
\hline 11 & 6,134 & 0,656 & 0,136 & 0,031 & 0,013 & 0,015 \\
\hline 12 & 3,896 & 1,133 & 0,054 & 0,026 & 0,021 & 0,023 \\
\hline $\begin{array}{l}\text { Moyenne : } \\
\mathrm{ng} / \mathrm{mL} \\
( \pm \mathrm{SD})\end{array}$ & $\begin{array}{c}7,482 \\
(4,312)\end{array}$ & $\begin{array}{c}0,614 \\
(0,261)\end{array}$ & $\begin{array}{c}0,067 \\
(0,049)\end{array}$ & $\begin{array}{c}0,029 \\
(0,018)\end{array}$ & $\begin{array}{c}0,010 \\
(0,006)\end{array}$ & $\begin{array}{c}0,011 \\
(0,005)\end{array}$ \\
\hline $\begin{array}{l}\mathrm{ng} / 3 \mathrm{~g} \text { de lait } \\
( \pm \mathrm{SD})\end{array}$ & $\begin{array}{c}149,640 \\
(86,240)\end{array}$ & $\begin{array}{c}12,280 \\
(5,220)\end{array}$ & $\begin{array}{c}1,340 \\
(0,980)\end{array}$ & $\begin{array}{c}0,580 \\
(0,360)\end{array}$ & $\begin{array}{c}0,200 \\
(0,120)\end{array}$ & $\begin{array}{c}0,220 \\
(0,100)\end{array}$ \\
\hline
\end{tabular}

Les résultats sont la moyenne de deux expériences, en double détermination chacune, pour la mesure des stéroïdes libres et conjugués. Les valeurs sont corrigées pour la perte survenue au cours des différentes étapes d'analyse et aussi pour les valeurs des bruits de fond. Les abréviations des hormones correspondent à celles de la figure 2. SD : déviation standard, ND : non détectable. * Les valeurs de l'estradiol ne tiennent pas compte de l'estradiol estérifié qui est de l'ordre de $14 \pm 2,5 \%$ en plus de l'estradiol quantifié.

Results are the means of two independent experiments in double determination for the measurement of free and conjugated steroids. Values are corrected for losses occuring during the different analysis steps and for the backgrounds. Abbreviations for hormones are in figure 2. SD: standard deviation, ND: non detectable. * The estradiol concentrations do not take into account esterified estradiol, which represents $14 \pm 2.5 \%$ in addition of the estradiol quantified.

trée (Slotin et al, 1970). Il est bien établi que la distribution des stéroïdes dans le lait dépend de la solubilité de chacun dans la partie aqueuse ou lipidique ; selon Heap et Hamon (1979), $80 \%$ de la progestérone se trouve dans la partie lipidique du lait.

Les stéroïdes en général, et la progestérone en particulier dans le lait et le plasma de femme, varient aussi quantitativement suivant l'état physiologique. La concentration de la progestérone dans le plasma des femmes augmente de $25 \mathrm{ng} / \mathrm{mL}$ en début de grossesse pour atteindre la valeur de 125 $\mathrm{ng} / \mathrm{mL}$ à la fin de cette période (Johansson, 1969). Selon Kulski et al (1977), la concentration en progestérone du lait de femme (mesurée par RIA) est de 110 à $148 \mathrm{ng} / \mathrm{mL}$ 18 jours avant l'accouchement, et chute au-dessous de $25 \mathrm{ng} / \mathrm{mL} 24$ heures après l'accouchement. Dans le lait des femmes collecté entre le $1,5^{\circ}$ et $30^{\circ}$ jour après la délivrance, par chromatographie en phase gazeuse couplée à la spectrométrie de masse, Sahlberg et Axelson (1986) identifient 
Tableau III. Comparaisons entre les laits de vache, de femme et les laits pour nourrissons. Comparisons between cow, women, and infant milks.

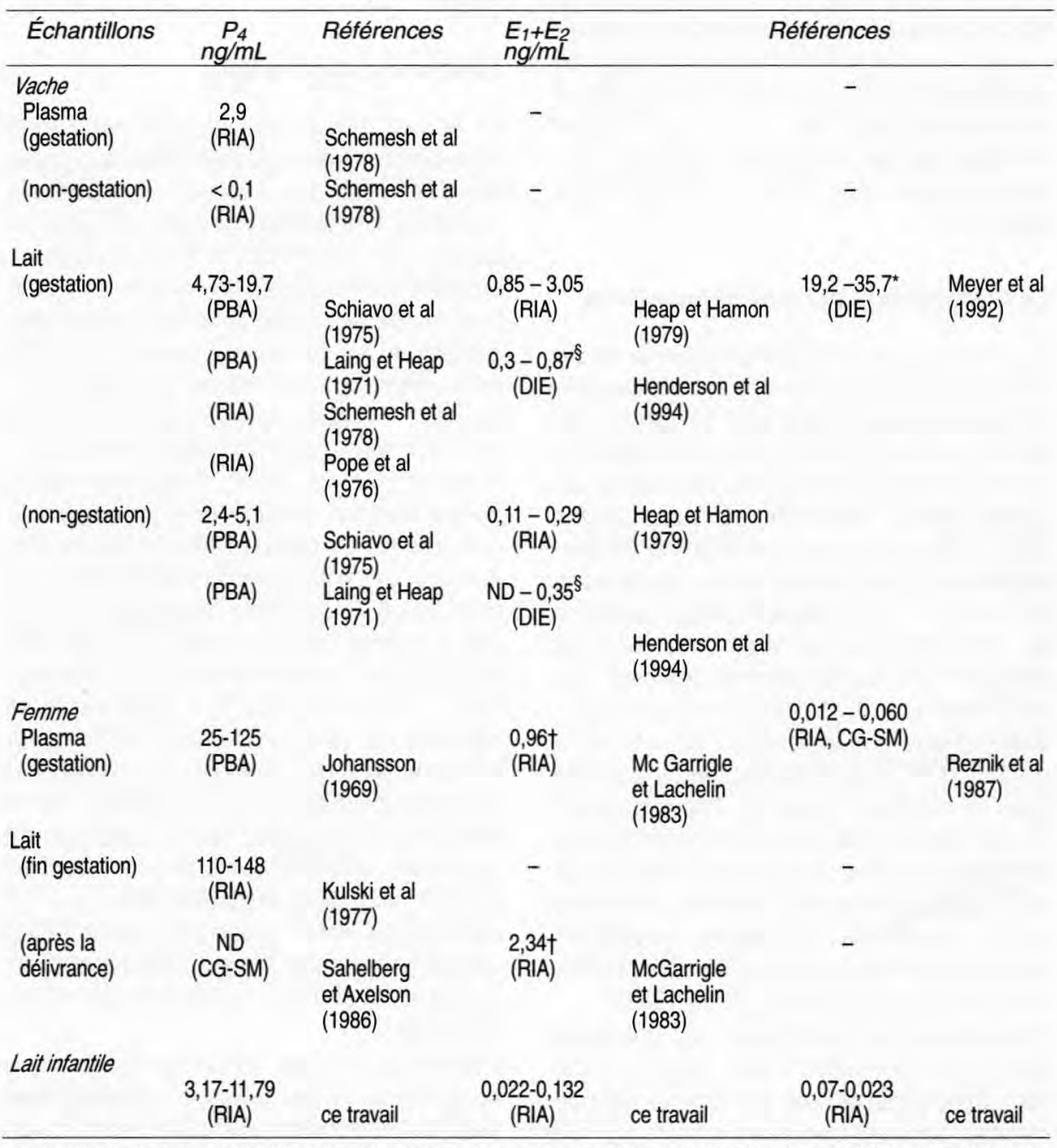

Les concentrations en stéroïdes libres plus conjugués sont comparées avec les données de la littérature (abréviations : voir figure 2) ; elles sont mesurées par différentes techniques : dosage radio-immunologique (RIA), protein binding assay (PBA), dosage immunoenzymatique (DIE), chromatographie en phase gazeuse - spectrométrie de masse (CG-SM). ND : non détectable. ${ }^{*}$ Ces valeurs ont été obtenues avant la parturition à partir d'échantillons d'urine. § Les valeurs rapportées le sont uniquement pour le sulfate d'estrone. † Les valeurs rapportées ont été obtenues chez des femmes allaitantes 3 à 5 jours après la délivrance.

The concentrations of free plus conjugated steroids are compared with the existing literature (for abbreviations see figure 2); they are measured by different techniques: radioimmunoassay (RIA), protein binding assay (PBA), enzymeimmunoassay (DIE), gaz chromatography / mass spectrometry (CG-SM). ND: non detectable. *These values were obtained before parturition from urine samples. § These values concern only estrone sulfate. $\dagger$ These values were obtained for lactating women 3 to 5 days after delivery. 
50 stéroïdes, y compris les $3 \beta$-hydroxy-5prégnènes, mais n'y détectent pas de progestérone (sauf dans un échantillon où elle était accompagnée de cortisol et de cortisone). Dans ce travail, la concentration de progestérone dans le lait commercialisé pour nourrissons $(7,48 \pm 4,31 \mathrm{ng} / \mathrm{mL}$ de lait reconstitué) est de l'ordre de celles publiées pour le lait de vache gestante (tableau III).

\section{La testostérone et l'androstènedione}

La concentration de la testostérone totale (libre et conjuguée) dans le lait des vaches non traitées est inférieure à $100 \mathrm{pg} / \mathrm{mL}$ pendant la phase estrale, mais cette concentration serait trois fois plus élevée pendant la phase lutéale (Hoffmann et Rattenberger, 1977). Chez la femme, ce stéroïde est peu sécrété par les ovaires ou les surrénales. Normalement, son taux représente moins de $10 \%$ des taux sécrétés chez l'homme (environ $10 \mathrm{mg} / \mathrm{j}$ ) par les cellules des Leydig (VanHelder et al, 1991). II n'y a pas d'étude, à notre connaissance, concernant la présence et la quantification de ce stéroïde dans le lait de femme. Or, l'accumulation de ce stéroïde dans le fluide des kystes mammaires à une concentration voisine de celle du plasma des mêmes patientes ( $473 \pm 459$ et $409 \pm 211 \mathrm{ng} / \mathrm{mL}$ respectivement) documente la possibilité de sa diffusion dans le lait (Bradlow et al, 1981).

Cependant, dans ce travail, sa présence avec une concentration moyenne de $29 \pm 18 \mathrm{pg} / \mathrm{mL}$ de lait reconstitué semble minime, sachant que dans le mois qui suit l'accouchement, plus de $95 \%(9,5 \mathrm{ng} / \mathrm{mL})$ des stéroïdes du lait des femmes sont des androgènes physiologiquement peu ou pas actifs par rapport à la testostérone : notamment de la déhydroépiandrostérone, de l'androstérone et de l'épiandrostérone (Sahlberg et Axelson, 1986). L'androstènedione est, après la progestérone, le stéroïde le plus abondant $(614 \pm 261 \mathrm{pg} / \mathrm{mL})$ présent dans les laits pour nourrissons. Sa présence est physiologique puisqu'elle peut être synthétisée à partir de la progestérone.

\section{L'estrone et l'estradiol}

Le sulfate d'estrone, sécrété par l'unité fœeto-placentaire, apparaît dans la circulation dès le début du second trimestre de la gestation. II présente chez les vaches gestantes une concentration dans le petit lait environ six fois plus élevée que le sulfate d'estradiol, alors que pour les vaches non gestantes, les concentrations de ces deux estrogènes sont du même ordre de grandeur (32-70 pg/mL pour le sulfate d'estrone et $15-54 \mathrm{pg} / \mathrm{mL}$ pour le sulfate d'estradiol ; Heap et Hamon, 1979). Plus récemment, en utilisant un dosage immunoenzymatique, Henderson et al (1994) ont démontré que les concentrations de sulfate d'estrone dans le lait des vaches gestantes $(297,4$ à $865,3 \mathrm{pg} / \mathrm{mL}$ ) sont également plus élevées que chez les non gestantes (de non détectable à $351,2 \mathrm{pg} / \mathrm{mL}$ ). II a également été rapporté par Heap et Hamon (1979) que la concentration des estrogènes (estrone et estradiol) conjugués dans le petit lait est en moyenne de $83 \mathrm{pg} / \mathrm{mL}$ chez les vaches non gestantes, et $2990 \mathrm{pg} / \mathrm{mL}$ chez les vaches au $238^{\circ}$ jour de la gestation, alors que les estrogènes libres présentent les mêmes concentrations dans le petit lait des vaches gestantes et non gestantes (environ $80 \mathrm{pg} / \mathrm{mL}$ ).

L'ensemble de ces résultats indique que les sulfates d'estrogènes en général, et le sulfate d'estrone en particulier, sont spécifiques de la gestation et leur présence dans le lait reconstitué apparaît donc logique. De plus, il a été rapporté par Maule Walker et al (1983) que les glandes mammaires de la vache (et de la brebis) sont capables de synthétiser de novo des estrogènes.

Plus récemment, Peaker et Taylor (1990) ont rapporté que les estrogènes sécrétés en fin de gestation par les glandes mammaires de la chèvre proviennent de l'aro- 
matisation locale des androgènes. La concentration de l'estrone totale (conjuguée et libre) que nous avons mesurée dans ce travail est environ sept fois plus importante que celle de l'estradiol total, ce qui correspond approximativement à la différence connue entre ces deux stéroïdes dans le lait des vaches gestantes.

Il est intéressant de noter que, dans le lait des femmes, l'estrone présente une concentration dix fois plus élevée que celle de l'estradiol, et que plus de $90 \%$ de ces deux stéroïdes se trouvent à l'état conjugué (McGarrigle et Lachelin, 1983). Cependant, les concentrations d'estrone et d'estradiol au total (hormones libres et conjuguées) mesurées dans ce travail $(22-132 \mathrm{pg} / \mathrm{mL})$ seraient au moins 18 fois plus faibles que les concentrations mesurées dans le lait des femmes (McGarrigle et Lachelin, 1983).

De plus, l'existence des stéroïdes sous forme estérifiée a été rapportée dans différents tissus, dont le sang (Hochberg et al, 1991). Il a été démontré que l'ester d'estradiol s'accumule essentiellement dans les tissus hydrophobes tels que la graisse, mais sa synthèse, notamment par des tumeurs du sein et par le foie bovin, a été confirmée (Hochberg et al, 1991). Cependant, la présence d'ester d'estradiol dans le lait n'a pas été, à notre connaissance, étudiée.

Les saponifications que nous avons réalisées sur ces échantillons de laits pour nourrissons montrent que les fractions non polaires écartées après délipidation contiennent $32,6 \pm 6,7 \mathrm{pg}$ d'estradiol estérifié pour $3 \mathrm{~g}$ de poudre de lait $(n=8)$. De plus, il existe une corrélation nette $(y=9,56$ $+0,91 x, r=1, p<0,01)$ jusqu'à $400 \mu \mathrm{L}$ d'échantillon correspondant à $1,2 \mathrm{~g}$ de poudre de laits, entre l'estradiol estérifié dosé et la quantité d'échantillon du dosage RIA (correspondant habituellement à $0,3 \mathrm{~g}$ de poudre / tube). L'estradiol estérifié représente ainsi, dans nos conditions, $14 \pm 2,5 \%$ de l'estradiol total quantifié (ester d'estradiol plus estradiol libre et conjugué).
Il a été rapporté par ailleurs que certains anticorps antiestradiol pourraient présenter des réactions croisées avec les phytoestrogènes (équol notamment) présents à des concentrations élevées dans certains laits de vaches (Barkley et al, 1985; Bannwart et al, 1988). Nos tests sur des gammes d'étalonnages en présence d'estradiol tritié et de concentrations croissantes d'équol indiquent que, à $50 \%$ de liaison, la réaction croisée des anticorps antiestradiol utilisés dans ce travail avec l'équol est négligeable $(<0,004 \%)$.

\section{La nortestostérone}

Selon Meyer et al (1992), la concentration de la nortestostérone totale (libre et glucurono-conjuguée), mesurée dans l'urine des vaches avant la parturition et par dosage immunoenzymatique, est de l'ordre de 19,2 à $35,7 \mathrm{ng} / \mathrm{mL}$. Cette concentration diminue dans les 2 jours qui suivent la naissance, et au $11^{\mathrm{e}}$ jour la nortestostérone devient indétectable.

Chez les femmes enceintes, la nortestostérone libre mesurée dans le plasma par RIA, ou par chromatographie en phase gazeuse puis spectrométrie de masse, varie de $12 \mathrm{pg} / \mathrm{mL}$ en début de grossesse jusqu'à $60 \mathrm{pg} / \mathrm{mL}$ à la fin. Ce stéroïde n'est pas détectable chez les femmes non enceintes, ni chez les hommes (Reznik et al, 1987). Du fait que la nortestostérone n'est détectable ni chez les vaches non gestantes ni chez les veaux âgés de 2 à 16 semaines, il a été suggéré que ce stéroïde est spécifique de la gestation, aussi bien chez les vaches que chez les femmes (Reznik et al, 1987 ; Meyer et al, 1992).

Dans ce travail, la présence de faibles concentrations de nortestostérone dans les poudres de laits (jusqu'à $23 \mathrm{pg} / \mathrm{mL}$ ) n'est donc pas surprenante. Ces valeurs sont de l'ordre des concentrations physiologiques chez les femmes enceintes. Pour Meyer et al (1992), c'est une concentration de $5 \mathrm{ng} / \mathrm{mL}$ dans l'urine de vache qui a été 
jugée comme un maximum au-delà duquel on considère qu'il y a fraude.

\section{Le diéthylstilbestrol et les $\beta$-agonistes}

Avec une sensibilité de $6,25 \mathrm{pg} / \mathrm{mL}$ de lait reconstitué, les résultats du dosage du diéthylstilbestrol ne permettent pas de détecter cette hormone dans les échantillons. Cette molécule ne semble pas être employée dans l'élevage des vaches laitières, tout du moins à un taux qui permettrait sa détection dans les laits maternisés.

Différentes techniques ont été développées ces dernières années pour la détection et le dosage des $\beta$-agonistes, notamment le clenbutérol, dans l'urine ou dans les tissus de bovins. La limite de détection varie selon la technique utilisée, mais aussi selon la nature de l'échantillon (Schmid et al, 1988). Chez l'homme, des concentrations de l'ordre de $1-2 \mathrm{ng} / \mathrm{mL}$ de clenbutérol dans l'urine sont détectables 48 heures après une injection en concentration thérapeutique ( $40 \mu \mathrm{g}$, Schmid et al, 1988). La présence de $\beta$-agonistes dans le lait de vache semble improbable : en effet, ces molécules pourraient être frauduleusement utilisées, mais préférentiellement chez des animaux destinés à la production de viande. Les concentrations homogènes mesurées pour les $\beta$-agonistes dans ce travail $(9,4 \pm 2,5 \mathrm{pg} / \mathrm{mL})$ sont sensiblement comparables aux bruits de fond et limites de détection de ces composés respectivement $(9,5 \pm 0,7$ et $12,5 \mathrm{pg} / \mathrm{mL})$. Comme pour les autres tests de surcharge, le test de surcharge des extraits avec des concentrations croissantes de clenbutérol montre des variations linéaires entre les concentrations ajoutées et celles mesurées seulement à partir du seuil de détection $(y=8,09+0,94 x, r=0,99, p<0,01)$.

En fait, c'est l'anticorps antisalbutamol qui a été utilisé pour le dosage des $\beta$-agonistes. Ainsi, les pourcentages de réactions croisées de cet anticorps pour $50 \%$ de liaison sont : avec le salbutamol de $100 \%$, brombutérol $193 \%$, clenbutérol $118 \%$, mabutérol $78 \%$, terbutaline $29 \%$, mapentérol $23 \%$, cimbutérol $17 \%$, cimatérol $12 \%$, tulobutérol $4 \%$, isoprotéranol $2 \%$ et pirbutérol $3 \%$ (laboratoire d'hormonologie, Marloie, Belgique). Les résultats montrent donc la non-détection des $\beta$-agonistes audessus de la limite de détection, dans tous les échantillons analysés.

\section{L'apport hormonal des poudres de laits}

Les concentrations de progestérone, d'estrone, d'estradiol, de testostérone et d'androstènedione dans le sang d'enfants des deux sexes âgés de 6 à 23 mois et pesant de 5 à $11 \mathrm{~kg}$, avec $75 \mathrm{~mL}$ de sang/kg (Hermann et Cier, 1969) sont de l'ordre de 100 , $11,9,7$ et $180 \mathrm{pg} / \mathrm{mL}$ respectivement (valeurs de référence de la Fondation de recherche en hormonologie, Fresnes, France). Pour un enfant prenant $500 \mathrm{~mL} / \mathrm{j}$ de lait reconstitué à $3 \mathrm{~g} / 20 \mathrm{~mL}$, la quantité de progestérone ingérée (environ $4 \mu \mathrm{g}$ ) correspond donc environ à 70 fois la quantité de ce stéroïde contenue dans le sang de ce même enfant. En revanche, pour la même quantité ingérée de lait maternel, il n'est pratiquement pas supposé absorber de progestérone. Par ailleurs, la quantité totale ingérée quotidiennement par l'enfant nourri au lait maternisé de l'ensemble des autres hormones (estrone, estradiol, testostérone et androstènedione, environ $0,36 \mu \mathrm{g}$ ) correspond environ à 3 fois le contenu en ces stéroïdes dans le sang de ce même enfant. On peut donc raisonnablement supposer qu'il absorbera notamment plus de progestérone en étant nourri au lait maternisé (de vache) que s'il était nourri au lait maternel. II semble cependant délicat de donner un pourcentage d'absorption de ces stéroïdes ingérés chez l'enfant, car ces pourcentages dépendent vraisemblablement en partie du régime et de la flore intestinale. 


\section{CONCLUSION}

D'une manière générale, les concentrations des stéroïdes qui ont été mesurées dans les poudres de laits sont de l'ordre de celles obtenues par différents auteurs dans le lait de vache. Les concentrations mesurées dans ce travail ne permettent donc pas de conclure à l'usage détectable des anabolisants recherchés dans le lait des vaches laitières, qui est utilisé pour fabriquer les poudres commercialisées pour nourrissons. La variété des anabolisants possibles, et le fait que la poudre de lait résulte d'un mélange, rendent cependant improbable la détection d'anabolisant, sauf en cas de traitement frauduleux grave sur un nombre important de vaches, et avec les mêmes hormones. Ainsi, ce type d'étude pourrait être répété régulièrement, les sources de lait étant nombreuses dans I'UE, et les conditions d'élevage pouvant être modifiées. D'autre part, nos dosages font apparaître une différence notable au niveau des constituants physiologiques hormonaux entre les laits maternisés et le lait de femme. En effet, alors que la progestérone se trouve en quantité importante dans le lait de vache et les laits pour nourrissons, le lait de femme après l'accouchement se caractérise par une absence de progestérone.

Sachant qu'une élévation significative de l'estradiol plasmatique du nourrisson a été observée après application de benzoate d'estradiol en pommade anti-inflammatoire sur le sein de femmes qui allaitent (professeur $\mathrm{P}$ Leymarie, communication personnelle) et que le système de détoxification et de métabolisation chez l'enfant n'est pas encore complètement développé, l'effet potentiel de l'apport hormonal des poudres de laits pour nourrissons mériterait d'être étudié, et sans négliger notamment les taux d'hormones estérifiées, dont les effets biologiques semblent parfois être prolongés par rapport à ceux des hormones libres elles-mêmes (Hochberg et al, 1991).

\section{REMERCIEMENTS}

Ce travail a été réalisé à la demande du Syndicat français des aliments de l'enfance et de la diététique (division lait infantiles), du Syndicat interprofessionnel des laits de conserve et d'Onilait sur des fonds de l'Union européenne, Contrat de recherche CEE 1001/90. Nous remercions les Drs $\mathrm{P}$ Leymarie, S Carreau et $\mathrm{P}$ Sourdaine pour leurs lectures critiques du manuscrit et surtout pour les informations apportées, le $\operatorname{Dr} C$ Benassayag pour l'aimable don d'équol, et Mme MJ Simon pour son assistance. Nous remercions également le Conseil général du Calvados pour son soutien financier.

\section{RÉFÉRENCES}

Axelson M, Sahlberg BL (1981) Solid extraction of steroid conjugates from plasma and milk. Anal Lett 14 , 771.782

Bannwart C, Adlercreutz $H$, Wähälä K, Kotiaho T, Hesso A, Brunow G, Hase T (1988) Identification of the Phyto-oestrogen 3',7-dihydroxisoflavan, an isomer of equol, in human urine and cow's milk. Biomed Environ Mass Spectrom 17, 1-6

Barkley MS, Lasley BL, Thompson MA, Shackleton CHL (1985) Equol: a contributor to enigmatic immunoassay measurement of estrogen. Steroids 46, 587-618

Bradlow HL, Rosenfeld RS, Kream J, Fleisher M, O'Connor J, Schwartz MK (1981) Steroid hormone accumulation in human breast cyst fluid. Cancer Res 41, 105-107

Girault J, Fourtillan JB (1990) Determination of clenbuterol in bovine plasma and tissues by gas chromatography-negative-ion chemical ionization mass spectrometry. J Chromatogr 518, 41-52

Heap RB, Hamon M (1979) Oestrone sulphate in milk as an indicator of viable conceptus in cows. $\mathrm{Br}$ Vet $J 135,355-363$

Henderson KM, Camberis M, Simmons MH, Starrs WJ, Hardie AHM (1994) Application of enzyme immunoassay to measure oestrone sulphate concentrations in cow's milk during pregnancy. I Steroid Biochem Mol Biol 50, 189-196

Hermann H, Cier JF (1969) Précis de physiologie I, Masson et Cie, Paris

Hochberg RB, Pahuja SL, Zielinsky JE, Larner JM (1991) Steroidal fatty acid esters. J Steroid Biochem $\mathrm{Mol}$ Biol 40, 577-585

Hoffmann B, Rattenberger E (1977) Testosterone concentrations in tissue from veal calves, bulls and heifers and in milk samples. J Anim Sci 46, 635-641

Johansson EDB (1969) Plasma levels of progesterone in pregnancy measured by a rapid competitive protein binding technique. Acta Endocrinol 61, 607-617

Kulski JK, Smith M, Hartman PE (1977) Perinatal concentrations of progestrone, lactose and $\alpha$-lactal- 
bumin in the mammary secretion of women. $J$ Endocrinol 74, 509-510

Laing JA, Heap RB (1971) The concentration of progesterone in the milk of cows during the reproductive cycle. Br Vet J 127, 19-21

Maule Walker FM, Davis AJ, Fleet IR (1983) Endocrine activity of the mammary gland: oestrogen and prostaglandin secretion by the cow and sheep mammary glands during lactogenesis. $\mathrm{Br}$ Vet J 139, 171-177

Meyer HHD, Falckenberg D, Janowski T, Rapp M, Rösel EF, van Look L, Karg H (1992) Evidence for the presence of endogenous 19-nortestosterone in the cow peripartum and in the neonatal calf. Acta Endocrinol 126, 369-373

McGarrigle HHG, Lachelin GCL (1983) Oestrone, oestradiol and oestriol glucosiduronates and sulphates in human puerperal plasma and milk. $J$ Steroid Biochem 18, 607-611

Peaker M, Taylor E (1990) Oestrogen production by the goat mammary gland: Transient aromatase activity during late pregnancy. $J$ Endocrinol 125, R1-R3

Pope GS, Majzlik I, Ball PJH, Leaver JD (1976) Use of progesterone concentrations in plasma and milk in the diagnosis of pregnancy in domestic cattle. $\mathrm{Br}$ Vet $J 132,497-506$
Reznik Y, Herrou M, Dehennin L, Lemarie M, Leymarie $P(1987)$ Rising plasma levels of 19-nortestosterone throughout pregnancy: Determination by radioimmunoassay and validation by gas chromatography - mass spectrometry. J Clin Endocrinol Metab 64, 1086-1088

Sahlberg BL, Axelson M (1986) Identification and quantification of free and conjugated steroids in milk from lactating women. J Steroid Biochem 25, 379-391

Schemesh M, Ayalon N, Shalev E, Nerya A, Schindler H, Milguir F (1978) Milk progesterone measurement in dairy cows: correlation with estrus and pregnancy determination. Theriogenology 9, 343-351

Schiavo JJ, Matuszczak RL, Oltenacu EB, Foote RH (1975) Milk progesterone in postpartum and pregnant cows as a monitor of reproductive status. J Dairy Sci 58, 1713-1716

Schmid J, Thomae K, Bücheler A (1988) A rapid liquidsolid extraction procedure for the quantification of clenbuterol in urine. Biomed Environ Mass Spectrom 17, 415-416

Slotin CA, Heap RB, Christiansen JM, Linzell JL (1970) Synthesis of progesterone by the mammary gland of the goat. Nature 225, 385-386

VanHelder WP, Kofman E, Tremblay MS (1991) Anabolic steroids in sport. Can J Sport Sci 16, 248-257 